\title{
Long-term efficacy and safety of internal neurolysis for trigeminal neuralgia without neurovascular compression
}

\author{
Andrew L. Ko, MD, ${ }^{1}$ Alp Ozpinar, BA, ${ }^{1}$ Albert Lee, MD, ${ }^{2}$ Ahmed M. Raslan, MD, ${ }^{1}$ \\ Shirley McCartney, PhD, ${ }^{1}$ and Kim J. Burchiel, MD ${ }^{1}$ \\ 1Department of Neurological Surgery, Oregon Health \& Science University, Portland, Oregon; and 2Department of Neurological \\ Surgery, University of Indiana, Indianapolis, Indiana
}

\begin{abstract}
OBJECT Trigeminal neuralgia (TN) occurs and recurs in the absence of neurovascular compression (NVC). While microvascular decompression (MVD) is the most effective treatment for TN, it is not possible when NVC is not present. Therefore, the authors sought to evaluate the safety, efficacy, and durability of internal neurolysis (IN), or "nerve combing," as a treatment for TN without NVC.
\end{abstract}

METHODS This was a retrospective review of all cases of Type $1 \mathrm{TN}$ involving all patients 18 years of age or older who underwent evaluation (and surgery when appropriate) at Oregon Health \& Science University between July 2006 and February 2013. Chart reviews and telephone interviews were conducted to assess patient outcomes. Pain intensity was evaluated with the Barrow Neurological Institute (BNI) Pain Intensity scale, and the Brief Pain Inventory-Facial (BPIFacial) was used to assess general and face-specific activity. Pain-free survival and durability of successful pain relief (BNI pain scores of 1 or 2) were statistically evaluated with Kaplan-Meier analysis. Prognostic factors were identified and analyzed using Cox proportional hazards regression.

RESULTS A total of 177 patients with Type 1 TN were identified. A subgroup of 27 was found to have no NVC on highresolution MRI/MR angiography or at surgery. These patients were significantly younger than patients with classic Type 1 TN. Long-term follow-up was available for 26 of 27 patients, and 23 responded to the telephone survey. The median follow-up duration was 43.4 months. Immediate postoperative results were comparable to MVD, with $85 \%$ of patients pain free and $96 \%$ of patients with successful pain relief. At 1 year and 5 years, the rate of pain-free survival was $58 \%$ and $47 \%$, respectively. Successful pain relief at those intervals was maintained in $77 \%$ and $72 \%$ of patients. Almost all patients experienced some degree of numbness or hypesthesia $(96 \%)$, but in patients with successful pain relief, this numbness did not significantly impact their quality of life. There was 1 patient with a CSF leak and 1 patient with anesthesia dolorosa. Previous treatment for TN was identified as a poor prognostic factor for successful outcome.

CONCLUSIONS This is the first report of IN with meaningful outcomes data. This study demonstrated that IN is a safe, effective, and durable treatment for TN in the absence of NVC. Pain-free outcomes with IN appeared to be more durable than radiofrequency gangliolysis, and IN appears to be more effective than stereotactic radiosurgery, 2 alternatives to posterior fossa exploration in cases of TN without NVC. Given the younger age distribution of patients in this group, consideration should be given to performing IN as an initial treatment. Accrual of further outcomes data is warranted.

http://thejns.org/doi/abs/10.3171/2014.12.JNS14469

KEY WORDS trigeminal neuralgia; internal neurolysis; microvascular decompression; neurovascular compression; gangliolysis; rhizotomy; functional neurosurgery; pain

$\mathrm{T}$ RIGEMINAL neuralgia (TN) is a pain syndrome characterized by severe, paroxysmal, lancinating facial pain. While many patients experience relief with carbamazepine or other drugs, many patients require surgery when their symptoms become refractory to medical therapy, or when they can no longer tolerate the medications. ${ }^{1}$ Surgical treatments such as neurectomy have targeted peripheral branches of the nerve external to the skull, and percutaneous ablative therapies have been directed at the gasserian ganglion using techniques such as radiofre-

ABBREVIATIONS BAEP = brainstem auditory evoked potential; BNI = Barrow Neurological Institute; BPI = Brief Pain Inventory; GKS = Gamma Knife surgery; HR = hazard ratio; IN = internal neurolysis; $\mathrm{MVD}$ = microvascular decompression; NVC = neurovascular compression; $P S R=$ partial sensory rhizotomy; $\mathrm{QOL}=$ quality of life; REZ = root entry zone; $\mathrm{RFL}=$ radiofrequency gangliolysis; $\mathrm{TN}=$ trigeminal neuralgia.

SUBMITTED March 5, 2014. ACCEPTED December 15, 2014

INCLUDE WHEN CITING Published online February 13, 2015; DOI: 10.3171/2014.12.JNS14469.

DISCLOSURE The authors report no conflict of interest concerning the materials or methods used in this study or the findings specified in this paper. 
quency gangliolysis (RFL), chemoablation using glycerol, or physical pressure using balloon microcompression, and stereotactic radiosurgery. Other procedures address the nerve within the posterior fossa, and can be nondestructive. In particular, microvascular decompression (MVD) has long been a mainstay of surgical treatment for TN. During this procedure, a vessel causing compression of the trigeminal nerve root entry zone (REZ) is freed from its arachnoid adhesions, moved away from the nerve, and held in place with Teflon pledgets. It is an effective, safe, nonablative procedure with durable results. ${ }^{1}$ Its success has resulted in the conflation of disease pathogenesis with neurovascular compression (NVC) of the trigeminal nerve at the REZ. ${ }^{6}$

However, not all patients with TN demonstrate NVC at the trigeminal REZ, and many asymptomatic patients do. In fact, as much as $17 \%$ of the general population shows NVC of the trigeminal nerve when imaged using highresolution MRI and MR angiography. Given an incidence of 1 per $10,000(0.01 \%)$ people for TN, $99.94 \%$ of individuals with NVC do not have TN. ${ }^{14}$ A recent large case series found that $28.8 \%$ of patients with Type $1 \mathrm{TN}$ exhibit no NVC. Moreover, the review of long-term recurrence rates of TN after successful MVD has been estimated at $4 \%-5 \%$ per year, which likewise occurs in the absence of NVC. 9 Therefore, NVC of the trigeminal nerve is not a necessary nor sufficient condition for the development or recurrence of $\mathrm{TN}$, which is clinically relevant. If no significant NVC of the trigeminal nerve exists, an MVD cannot be performed. The advent of high-resolution brainstem, nerve, and vascular structure imaging has made it possible to ascertain with a very high level of certainty (96\% sensitivity, 90\% specificity) whether NVC is present prior to any invasive procedure. ${ }^{9}$ In the absence of NVC on imaging, the question arises as to whether to recommend a posterior fossa exploration versus a peripheral or percutaneous ablative therapy.

In the case of negative posterior fossa exploration, partial sensory rhizotomy (PSR) is a possible treatment option. This procedure entails division of the lateral one-half to two-thirds of the sensory root, $2-5 \mathrm{~mm}$ from the pons. In contrast to MVD, PSR is an ablative procedure, and therefore results in sensory deficits. The failure rate for PSR at 1 year has been reported to be as high as $42 \%$, with an additional $2 \%$ per year thereafter. ${ }^{21}$ Patient satisfaction rates after this procedure are significantly less than for MVD. ${ }^{22}$

There is another option for treating $\mathrm{TN}$ in which no NVC is observed on imaging or during surgery. We refer to this procedure as internal neurolysis (IN), also called nerve "brushing" or "combing." Internal neurolysis is a procedure in which all or portions of the trigeminal nerve are divided longitudinally along its fibers between the pons and the porus trigeminus. Thus, this nomenclature is not strictly accurate, as internal neurolysis entails separation of nerve fascicles; the internal epineurium, which invests these fascicles, derives from the invagination of arachnoid and dura as the nerve exits the dura, and hence does not exist where the IN is performed intradurally. However, this term is more descriptive than more colloquial references to "brushing" or "combing." This procedure differs from PSR in that nerve fibers are not cut; thus sensory deficits may be less severe.

Internal neurolysis has been described previously in the literature. ${ }^{711}$ However these studies do not report outcomes using Kaplan-Meier statistics or summary measures of outcome events over time. Thus, the interpretation of these results is difficult. To our knowledge, this is the first report of long-term IN outcomes in patients with TN without NVC.

\section{Methods}

\section{Study Design and Diagnostic Criteria}

A retrospective review was undertaken of patients 18 years of age or older with Type 1 or Type 2 TN treated at Oregon Health \& Science University from July 2006 to February 2013. The study was approved by the institutional review board of Oregon Health \& Science University.

Diagnosis was made using a computer-based questionnaire as well as clinical interview and examination., ${ }^{9,12,13}$ Using the Burchiel classification of facial pain, all patients reviewed for this study had Type $1 \mathrm{TN}$, characterized by idiopathic, episodic, lancinating pain lasting several seconds, with pain-free intervals between attacks. Importantly, these patients did not have Type $2 \mathrm{TN}$, which is predominantly ( $>50 \%$ of the time) aching, throbbing, or burning pain with a minor component of lancinating pain. This is a clinical diagnosis, and hence all diagnoses were made during initial evaluation, prior to advanced imaging, and prior to findings during surgery. Using the International Classification of Headache Disorders (third edition), these patients would have been classified as having classic TN, or classic TN with concomitant persistent pain. ${ }^{5}$ A phone survey and chart review were completed to assess preoperative neurological status, previous treatments for TN, and surgical outcome. Preoperative presence or absence of sensory deficit, defined as hypesthesia to pinprick, was documented in charts for all patients.

All patients underwent evaluation with high-resolution MRI/MR angiography, and these images were reconstructed in 3D and evaluated for NVC as previously described $^{14}$ (Fig. 1A).

\section{Surgical Procedure}

All surgical procedures were performed by the senior author (K.J.B.). Patients are placed supine with the affected side up, the ear positioned parallel to the floor, and the chin flexed. Baseline brainstem auditory evoked potentials (BAEPs) were obtained. BAEPs and facial nerve electromyography were monitored throughout the surgery. A linear incision is made $0.5 \mathrm{~cm}$ posterior to the digastric groove, starting at the top of the pinna and extending to the mastoid tip. A $3 \times 3-\mathrm{cm}$ retrosigmoid craniectomy is performed using a high-speed drill and Kerrison rongeurs to expose the transverse-sigmoid junction. Any exposed mastoid air cells are carefully waxed. The dura is opened in a U-shaped fashion along the inferior border of the transverse sinus and the posterior border of the sigmoid sinus. The anterior and superior edges of this opening are reflected upwards and secured with 4-0 braided nylon and CSF egress is encouraged to relax the cerebellum. A cop- 

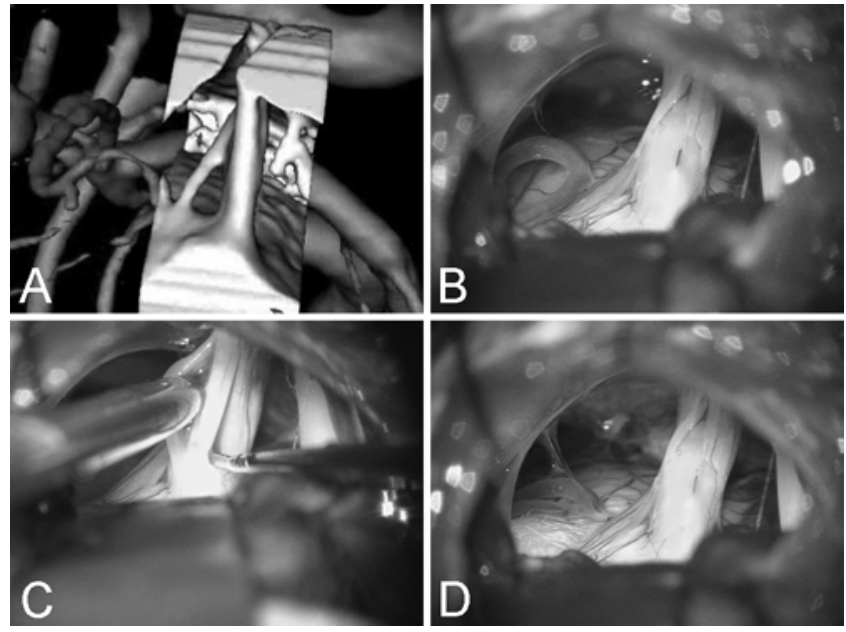

FIG. 1. Internal neurolysis: imaging and intraoperative views. A: Reconstruction in $3 \mathrm{D}$ of high-resolution MRI/MR angiography, showing relationships between the vasculature, pons, and trigeminal nerve. A branch of the superior cerebellar artery lies adjacent to the portio minor, causing no significant compression of the trigeminal nerve REZ. B: Intraoperative view analogous to panel A, showing no NVC. C: The IN procedure entails separating the nerve longitudinally, using a blunt-tip dissector. The nerve is divided into 8-10 bundles from the pons to the petrous bone. D: Intraoperative view showing the final configuration of the nerve. A small Teflon pledget has been placed between the superior cerebellar artery and the brainstem.

per brain retractor is advanced along the superior surface of the cerebellum and the cisterna are opened under direct microscopic visualization. The superior petrosal veins are coagulated and cut, and arachnoid adhesions are lysed to explore the cerebellopontine angle. The trigeminal nerve and REZ are carefully examined for NVC by an artery or vein. When none is found (Fig. 1B), the trigeminal nerve itself is then longitudinally divided along its fibers, using a straight blunt-tip bayonet probe, into 5-10 bundles from the REZ to the petrous bone (Fig. 1C and D). We include both the portio minor and portio major in the neurolysis, as the portio minor has been shown to carry unmyelinated fibers implicated in pathological facial pain, and neural anastomoses exist between these components of the trigeminal nerve. ${ }^{19}$ Hemostasis is ensured. A watertight closure of the dura is performed and augmented with only collagen matrix and fibrin glue. Any exposed mastoid air cells are rewaxed, and a cranioplasty is performed with self-setting calcium hydroxyapatite cement. Closure of muscle, soft tissue, and skin is performed in layers in a standard fashion.

\section{Outcome Measures}

Surgical outcomes for IN were scored using the Barrow Neurological Institute (BNI) Pain Intensity Scale. ${ }^{16}$ Complete pain relief was defined as patients who were painfree without medications. A single episode of lancinating pain constituted treatment failure, as well as the return of any constant or aching pain present before IN. The procedure was considered a success in patients with no or occasional pain, without medications (BNI scale score of 1 or 2). Recurrence was defined as transition from pain-free to good response, or from success to failure (BNI scale score of 3 or greater).

Patients were specifically asked to rate the effect that facial numbness had on their daily activities. Quality of life (QOL) was evaluated using elements of the Brief Pain Inventory-Facial (BPI-Facial) that address interference with general activities and face-specific activities (Table 1). The internal reliability and construct validity of this questionnaire has been confirmed. ${ }^{4}$ Additional questions were directed toward the frequency and intensity of pain, and the use of medications, and used to derive the BNI score. This questionnaire was administered by an independent observer not involved in patient care (A.O.). Chart review was performed by personnel who were not involved in initial patient evaluation or the IN procedure.

\section{Prognostic Factors}

We examined the following patient- and neuralgiarelated factors: sex, age, presence of sensory deficit, and presence of concomitant persistent pain prior to surgery. Exposure to previous procedures to treat TN was also examined as a prognostic factor.

\section{Statistical Analysis}

Data analysis was performed using commercially available software (MATLAB). Kaplan-Meier survival analysis was performed and log-rank tests were used to compare survival curves. The Yates correction was applied due to small sample size. Risk factors were evaluated using the Cox proportional hazards method. BPI-Facial scores were compared using the student t-test and the Wilcoxon rank-

TABLE 1. The BPI-Facial scale*

\begin{tabular}{c}
\hline \multicolumn{1}{c}{ Variable } \\
\hline Pain intensity \\
\hline Current pain \\
Worst pain in last wk \\
\hline Least pain in last wk \\
\hline Average pain in last wk \\
\hline Interference in general activities \\
\hline General activity \\
\hline Mood \\
\hline Walking ability \\
\hline Normal work \\
\hline Relations w/ other people \\
\hline Sleep \\
\hline Enjoyment of life \\
\hline Interference in face-specific activities \\
\hline Eating a meal \\
\hline Touching one's face \\
\hline Brushing or flossing teeth \\
\hline Smiling or laughing \\
\hline Talking \\
\hline Opening one's mouth widely \\
\hline Eating hard foods \\
\hline
\end{tabular}

* Each item scored from 1 to 10. 
sum test, and correlation was calculated in the usual fashion. Those $\mathrm{p}$ values $<0.05$ were considered statistically significant.

\section{Results}

\section{Demographics and Clinical Characteristics}

Twenty-seven patients were identified as having undergone an IN for Type $1 \mathrm{TN}$ in the time period described; of these 27 patients, 7 were men (26\%; Table 2). The average age of the patients was 46.9 years, with a median age of 48.5 years (range 25.7-71.3 years). Notably, the age distribution of the patients with Type $1 \mathrm{TN}$ and no NVC differed from that of patients with Type $1 \mathrm{TN}$ with NVC ( $p=0.004,2$-sample Kolmogorov-Smirnov test). The age distribution for patients undergoing IN was bimodal, with 1 peak at age 32 years, and 1 peak at 53 years. In contrast, patients with Type $1 \mathrm{TN}$ and NVC were older, with a peak age of 61 years (Fig. 2).

Pain treated with IN was right-sided in 13 patients and left-sided in 14 patients. The TN divisions that were affected are detailed further in Table 2. Four patients (15\%) had documented sensory deficits prior to intervention, all of whom underwent previous ablative treatment for $\mathrm{TN}$; all other patients had no hypesthesia in the distribution of their pain. Twelve patients (44\%) presented with a component of constant or aching pain. Ten patients had a previous treatment for Type 1 TN, including MVD, Gamma

TABLE 2. Patient demographics and clinical characteristics

\begin{tabular}{|c|c|}
\hline Variable & Value \\
\hline Total no. of patients & 27 \\
\hline Follow-up, w/ notes & 26 \\
\hline Follow-up, telephone survey & 23 \\
\hline Males & 7 \\
\hline Females & 20 \\
\hline \multicolumn{2}{|l|}{ Age (yrs) } \\
\hline Mean & 46.9 \\
\hline Median & 48.5 \\
\hline Range & $25.7-71.3$ \\
\hline Sensory disturbance & 4 \\
\hline Concomitant persistent pain & 12 \\
\hline \multicolumn{2}{|l|}{ Laterality } \\
\hline $\mathrm{Rt}$ & 12 \\
\hline $\mathrm{Lt}$ & 14 \\
\hline \multicolumn{2}{|l|}{ TN divisions } \\
\hline V1 & 1 \\
\hline V2 & 2 \\
\hline V3 & 4 \\
\hline $\mathrm{V} 1 / \mathrm{V} 2$ & 2 \\
\hline V2/V3 & 8 \\
\hline V1/V2/V3 & 9 \\
\hline \multicolumn{2}{|c|}{ Previous treatments (no. of patients) } \\
\hline MVD & $8(6)$ \\
\hline GKS & $5(4)$ \\
\hline RFL & $2(1)$ \\
\hline
\end{tabular}

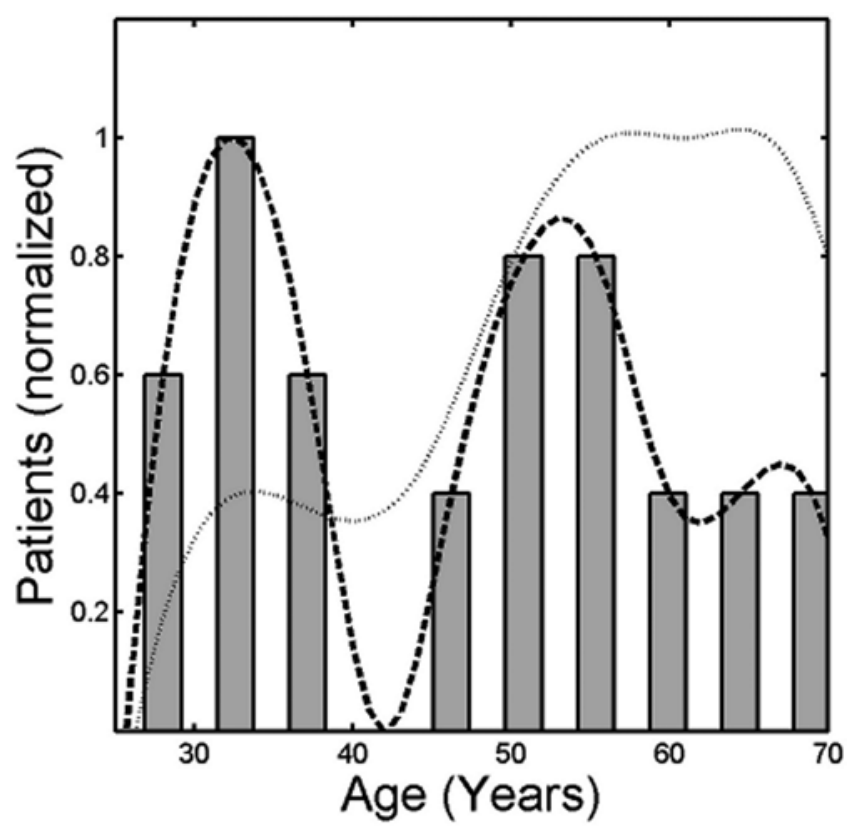

FIG. 2. Normalized histogram representing the age distribution of patients undergoing IN compared with all patients with Type $1 \mathrm{TN}$ and NVC. Gray bars denote patients in this study. Note the bimodal distribution (dashed line), with a subset of patients with IN peaking at age 32, and an older IN group at age 53 . Both peaks are significantly younger than the most common age for patients with Type 1 TN and NVC (dotted line), which peaks at age 61. Curves were generated using cubic spline interpolation, and all histogram values were normalized by the most common age bin in each group.

Knife surgery (GKS), RFL, or some combination of the 3 (Table 2). Clinical follow-up was possible for 26 of 27 patients, with details presented below.

\section{Imaging and Operative Findings}

All patients underwent preoperative high-resolution MRI/MR angiography to assess for NVC. No patients undergoing IN were found to have definite NVC intraoperatively. Two patients in whom NVC was suspected on imaging did not have compression during surgical exploration. One patient had a small petroclival meningioma abutting the porus trigeminus without compression of the trigeminal REZ. Six patients had previously undergone MVD procedures, and all TNs were found to be adequately decompressed at the time of IN. One patient had a small vein abutting the REZ. These 6 patients had Teflon pledgets placed between vascular structures and the trigeminal nerve in addition to their IN.

\section{Follow-Up}

The average follow-up duration was 39.1 months, with a median follow-up of 43.4 months (range 15.0-66.6 months). One patient was lost to follow-up due to death from other causes and was not included in any statistical analyses. There were no other deaths during the study period. Three patients declined to participate in the phone survey or were unreachable for follow-up. In these cases, the last clinic note was used to determine the BNI score and censored. BPI-Facial scores were available for 23 pa- 
tients. A total of $16(62 \%)$ of 26 patients underwent follow-up for longer than 3 years, and all of these patients responded to the telephone survey.

\section{Immediate Postoperative Outcomes}

Initial results for IN were quite favorable, with $22(85 \%)$ of 26 patients reportedly pain free after surgery, and 25 (96\%) of 26 patients having only occasional pains without medication.

\section{Long-Term Outcomes}

Long-term outcomes were examined using KaplanMeier analysis. We examined complete and successful pain relief as defined above. Successful response to IN was maintained in $77 \%$ of patients at 1 year, and $72 \%$ at both 2 and 5 years. The median time to failure was not reached within the follow-up period of this study. Painfree survival at 1,2, and 5 years was $58 \%, 52 \%$, and $47 \%$, respectively. Median pain-free survival was 24.8 months (Fig. 3). A summary of treatment event data over time is presented in Table 3.

Univariate analysis of prognostic factors was performed. Two models were examined, one to account for time to treatment failure, and one to account for time to return of any pain at all. The only significant effect was noted in conjunction with previous treatment for $\mathrm{TN}$ with MVD, GKS, or RFL, which led to decreased good response to treatment $(\mathrm{p}=0.006$, log-rank test with Yates correction). Ten of 26 patients were included in this group. For these patients, treatment rapidly failed, with a median recurrence time of 8.7 months. After 1 year, the success rate of IN in these patients was only $40 \%$, but remained stable, with good response maintained in 4,4 , and 1 patients through 2, 3, and 5 years, respectively. In contrast, patients without a previous treatment for TN exhibited a $94 \%$ success rate after 1 year, with no further failures throughout the follow-up period (Fig. 3). Previous treatment for TN did not have a statistically significant effect on pain-free survival after IN ( $p=0.559$, log-rank test with Yates correction). Notably, all previously treated patients who did not achieve partial pain relief $(60 \%)$ had some degree of pain recurrence within 4 months (Table 4).

Cox proportional hazards analysis was performed as well. Due to the small sample size, we did not examine the effect of preoperative neurological deficit, and included 4 factors instead of 5 in our models (Table 4). As above, previous treatment for $\mathrm{TN}$ was the only factor that had a significant impact on outcome (hazard ratio [HR] 2.75, $\mathrm{p}$ $=0.02$ ).

\section{Further Interventions}

Seven patients experienced recurrence of their Type 1 TN. Of these 7 patients, 3 underwent further treatment, all with RFL. One patient underwent RFL twice, for a total of 4 subsequent interventions for recurrent Type $1 \mathrm{TN}$. At the time of the phone questionnaire, 2 of the 3 patients were pain free. The 1 patient refractory to treatment was subsequently found to not have TN, developing bilateral pain outside the initial and treated distribution (and outside the distribution of the trigeminal nerve).
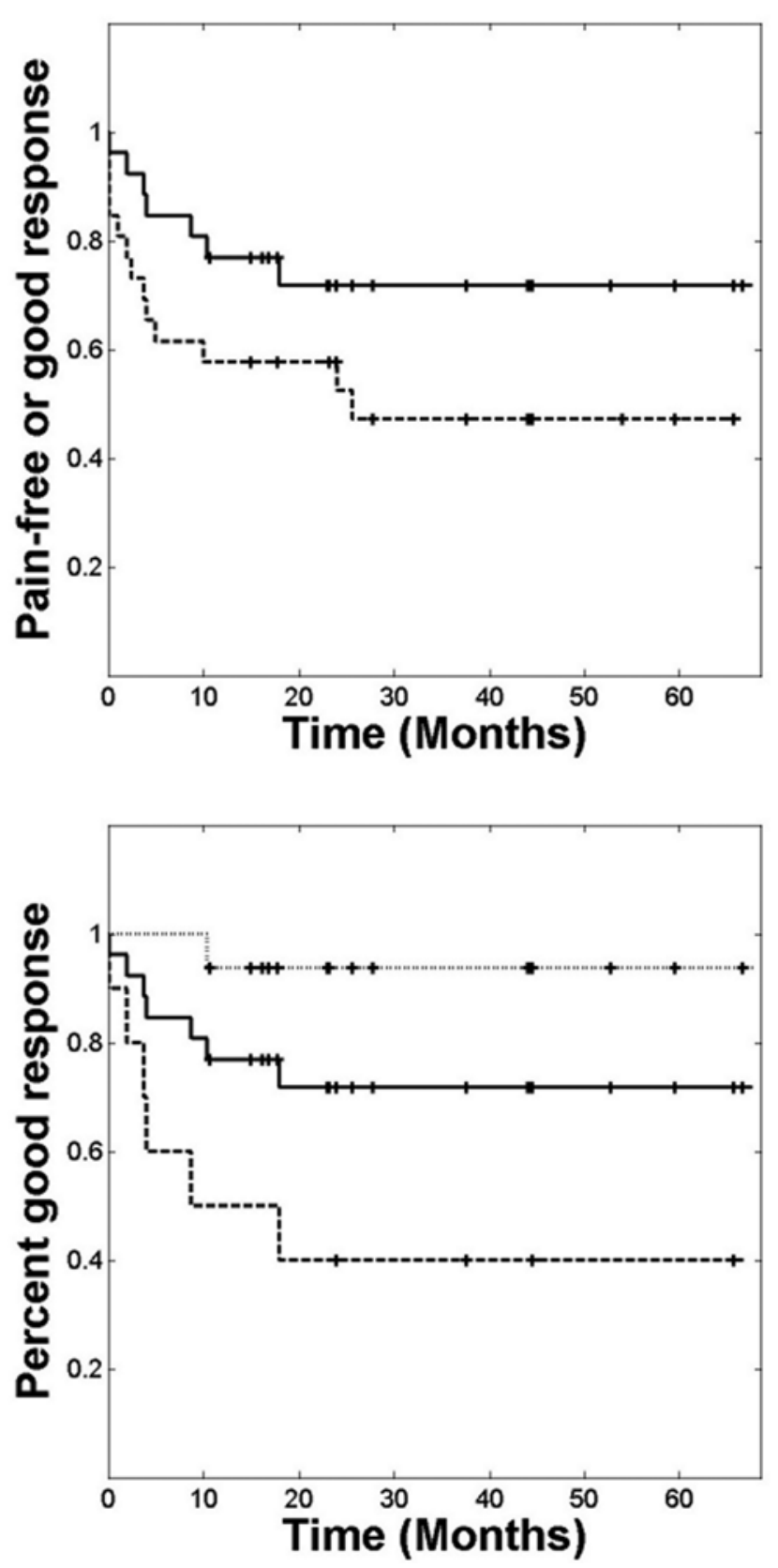

FIG. 3. Kaplan-Meier survival curves. Upper: Survival curves for complete and successful pain relief. The dashed line represents pain-free survival rates, with recurrence defined as the return of any pain. The solid line represents treatment failure rates, with treatment failure defined as more than occasional pain, or any pain requiring treatment with medication. Censored data are represented as hatch marks. Lower: Effect of previous treatment for TN on treatment failure rates. The solid line is the same as above, whereas the dashed line represents failure rates in patients with previous treatment for TN. The dotted line represents only patients for whom IN was the initial treatment. As above, hatch marks denote censored data points. There was a significant difference between previously treated and nontreated patients $(p=0.006$, log-rank test with Yates correction).

\section{Immediate Operative Complications}

There was no perioperative mortality. One CSF leak was noted in the immediate postoperative period and repaired definitively on postoperative Day 7. No intracranial 
TABLE 3: Survival with good response after internal neurolysis

\begin{tabular}{lrrrrrrr}
\hline Outcome & $1 \mathrm{Mo}$ & $6 \mathrm{Mos}$ & $1 \mathrm{Yr}$ & 2 Yrs & 3 Yrs & 4 Yrs & 5 Yrs \\
\hline Success & 25 & 23 & 20 & 12 & 10 & 6 & 3 \\
\hline Failure & 1 & 3 & 6 & 7 & 7 & 7 & 7 \\
\hline Censored & 0 & 0 & 0 & 7 & 9 & 13 & 16 \\
\hline Pain free & 22 & 16 & 15 & 11 & 8 & 3 & 1 \\
\hline Failure & 4 & 10 & 11 & 11 & 12 & 12 & 12 \\
\hline Censored & 0 & 0 & 0 & 4 & 6 & 11 & 13 \\
\hline
\end{tabular}

hemorrhage was noted on postoperative CT. No symptomatic infarcts were noted. There were no surgical-site infections. There were no cases with weakness of the muscles of mastication recorded postoperatively. All BAEPs were noted to be at baseline levels at the end of each procedure, and no temporary or permanent hearing loss resulted. There were no extraocular muscle or facial palsies. The immediate postoperative complication rate was $3.8 \%$.

\section{Numbness}

All patients except for 1 (96\%) reported subjective numbness or hypesthesia in the face after IN. Objective sensory examination was not performed. We sought to evaluate the impact of this numbness on QOL using elements of the BPI-Facial score. Average scores for each patient were strongly correlated with BNI ratings (Fig. 4 upper) for both face-specific activities (BPI-Facial) and general impact on life (BPI-General) scores (rho $=0.84$ and $0.89, \mathrm{p}=0.00$ and 0.00 [Spearman's rank correlation], for BPI-Facial and BPI-General, respectively). However, values for BPI scores were non-normal and heavily rightskewed across all patients. Thus, we used Wilcoxon's ranksum test as well, to compare median values between these groups, rather than only mean scores. Mean and median BPI scores were significantly lower in patients with good responses using 2-sample t-tests (BPI-Facial success/failure $=1.64 / 5.77, \mathrm{p}=0.00 ; \mathrm{BPI}-$ General success/failure $=$ $1.15 / 5.19, \mathrm{p}=0.00)$ and Wilcoxon's rank-sum tests (BPIFacial success/failure $=1.31 / 5.4, \mathrm{p}=0.002 ; \mathrm{BPI}-$ General success/failure $=1.00 / 4.7, \mathrm{p}=0.00$ ).

The presence of significant pain confounds the impact of numbness on patient QOL. Hence, we compared BPI scores for patients with numbness only, and patients with only occasional pain (BNI score of 1 vs 2). There was no difference in mean scores between these groups, as shown in Fig. 4 lower (BPI-Facial, $\mathrm{p}=0.14$; BPI-General, $\mathrm{p}=$ $0.48)$. There was no significant difference between median BPI scores for patients with pain-free outcomes and good outcomes (BPI-Facial pain free $/$ good $=1.36 / 1.29$, $\mathrm{p}=0.8166$; BPI-General pain free/good $=1.00 / 1.00 ; \mathrm{p}=$ $0.3431)$.

\section{Trigeminal Deafferentation Pain}

In total, 7 of 23 patients with telephone questionnaire follow-up maintained that they had constant, aching pain after surgery. However, 2 of these patients had pain that was outside the distribution of the treated trigeminal nerve: 1 patient developed bilateral facial pain, while the other reported pain in the face and ear that radiated to the shoul-
TABLE 4: Univariate and multivariate analysis of prognostic factors for time to treatment failure and time of pain-free survival

\begin{tabular}{|c|c|c|c|c|}
\hline Variable & Mos & $\mathrm{p}$ Value & $\mathrm{HR}$ & $p$ Value \\
\hline \multicolumn{5}{|c|}{ Median time to failure } \\
\hline \multicolumn{5}{|c|}{ Age (yrs) } \\
\hline$>42$ & 24.8 & 0.780 & 0.02 & 0.682 \\
\hline$<42$ & 16.8 & & & \\
\hline \multicolumn{5}{|l|}{ Sex } \\
\hline Male & 10.4 & 0.267 & 1.892 & 0.061 \\
\hline Female & 23.4 & & & \\
\hline \multicolumn{5}{|c|}{ Concomitant persistent pain } \\
\hline Present & 17.9 & 0.601 & 1.615 & 0.102 \\
\hline Not present & 44.1 & & & \\
\hline \multicolumn{5}{|c|}{ Previously treated TN } \\
\hline Present & 8.7 & $0.006^{*}$ & 2.753 & $0.018^{*}$ \\
\hline Not present & 24.4 & & & \\
\hline \multicolumn{5}{|l|}{ Preop deficit } \\
\hline Present & 22.9 & 0.710 & & \\
\hline Not present & 23.0 & & & \\
\hline \multicolumn{5}{|l|}{ Pain-free survival } \\
\hline \multicolumn{5}{|l|}{ Age (yrs) } \\
\hline$>42$ & 24.8 & 0.503 & -0.01 & 0.708 \\
\hline$<42$ & 10.0 & & & \\
\hline \multicolumn{5}{|l|}{ Sex } \\
\hline Male & 2.0 & 0.287 & 0.48 & 0.774 \\
\hline Female & 20.4 & & & \\
\hline \multicolumn{5}{|c|}{ Concomitant persistent pain } \\
\hline Present & 4.4 & 0.356 & 0.79 & 0.839 \\
\hline Not present & 24.1 & & & \\
\hline \multicolumn{5}{|c|}{ Previously treated TN } \\
\hline Present & 3.7 & 0.559 & 0.865 & 0.606 \\
\hline Not present & 23.6 & & & \\
\hline \multicolumn{5}{|l|}{ Preop deficit } \\
\hline Present & 14.0 & 0.953 & & \\
\hline Not present & 17.7 & & & \\
\hline
\end{tabular}

* Statistically significant.

der. Of the patients with pain in a trigeminal distribution, 4 of 5 patients reported aching pain in the same distribution preoperatively. The incidence of new aching pain in an area of numbness occurred in $1(4 \%)$ of 23 patients. Notably, this patient presented with numbness preoperatively. All patients with any deafferentation pain $(5 / 23,22 \%)$ had undergone a previous procedure to treat $\mathrm{TN}$.

\section{Discussion}

This study shows that IN is a safe and efficacious treatment for TN with or without concomitant persistent pain when there is no NVC found on imaging or at surgery. The mechanism by which IN produces pain relief is unclear. Internal neurolysis is a destructive procedure in comparison with MVD. It appears to produce at least a subjective numbness in nearly all patients. Therefore, a putative mechanism for its effectiveness is the reduction of trig- 
gering of lancinating pain in Type $1 \mathrm{TN}$, much like RFL, PSR, and other ablative procedures. Unlike RFL or PSR, however, IN as performed at our institution covers all distributions of the trigeminal nerve. This may contribute to its effectiveness and durability compared with RFL in particular. At least anecdotally, stimulation in one distribution can cause pain in another; touching the cheek or forehead can trigger lancinating pain in the jaw, for example. Internal neurolysis may disrupt interconnections between nerve fibers communicating between distributions of the trigeminal nerve prior to entrance into the brainstem. Further study is warranted.

Nevertheless, this procedure resulted in immediate relief of all pain in $85 \%$ of patients and significant pain relief in $96 \%$ of patients. The success rate is durable, with an estimated $72 \%$ of patients maintaining significant pain relief without medications at 5 years. Previous treatment for TN was identified as a poor prognostic factor for successful treatment, with a median time to treatment failure of 8.7 months in those treated with MVD, GKS, or RFL, and a median time to failure of 24.4 months in those without previous treatment. This was statistically significant in both univariate and multivariate analysis.

The immediate results are comparable to those for MVD. The largest series reported that $82 \%$ of patients were pain free immediately after MVD, with partial relief in 98\%.' Results for MVD in the long term indicate that this nonablative treatment appears to be more durable; at 1 year, $75 \%$ of patients are pain free and $84 \%$ experience partial relief; this is in contrast to $58 \%$ and $77 \%$ for pain free and partial relief after IN, respectively. Interestingly, in cases of reoperation for TN, MVD showed approximately a $50 \%$ pain-free rate at 5 years, which is very similar to success rates we find for IN at that time point. ${ }^{1}$

In the small number of patients requiring retreatment, RFL remains a viable option after IN. This is especially important given the age distribution of patients without NVC. In this study, the patient age distribution was bimodal, with a significantly younger patient population presenting with Type $1 \mathrm{TN}$ and no NVC.

\section{Comparison With Other Ablative Therapies for TN}

There is no question that MVD remains the preferred treatment for TN with NVC. However, given that MVD is not an option for patients without NVC, the long-term success rate for IN suggests that it is a good option for treatment of $\mathrm{TN}$ in these cases. Other treatment options that are widely used in this scenario include PSR, RFL, and GKS. We sought to compare, at least qualitatively, our results with IN to these alternatives.

Outcomes for pain-free survival and long-term pain relief are not dissimilar to those reported for PSR. In a series of 83 patients undergoing PSR for no or inadequate NVC, $48 \%$ of patients were immediately pain free, with an additional $22 \%$ having some improvement in their pain. The rate of significant pain recurrence at 1 year was $17 \%$. Pain recurrence of any sort at 1 year was $42 \%$. Thereafter, failure rates for both measures were roughly $2 \%$ per year; after 5 years, roughly $70 \%$ of remaining treatment successes continued to be pain free, and $50 \%$ had significant reduction of pain. ${ }^{21}$ Other long-term follow-up studies of
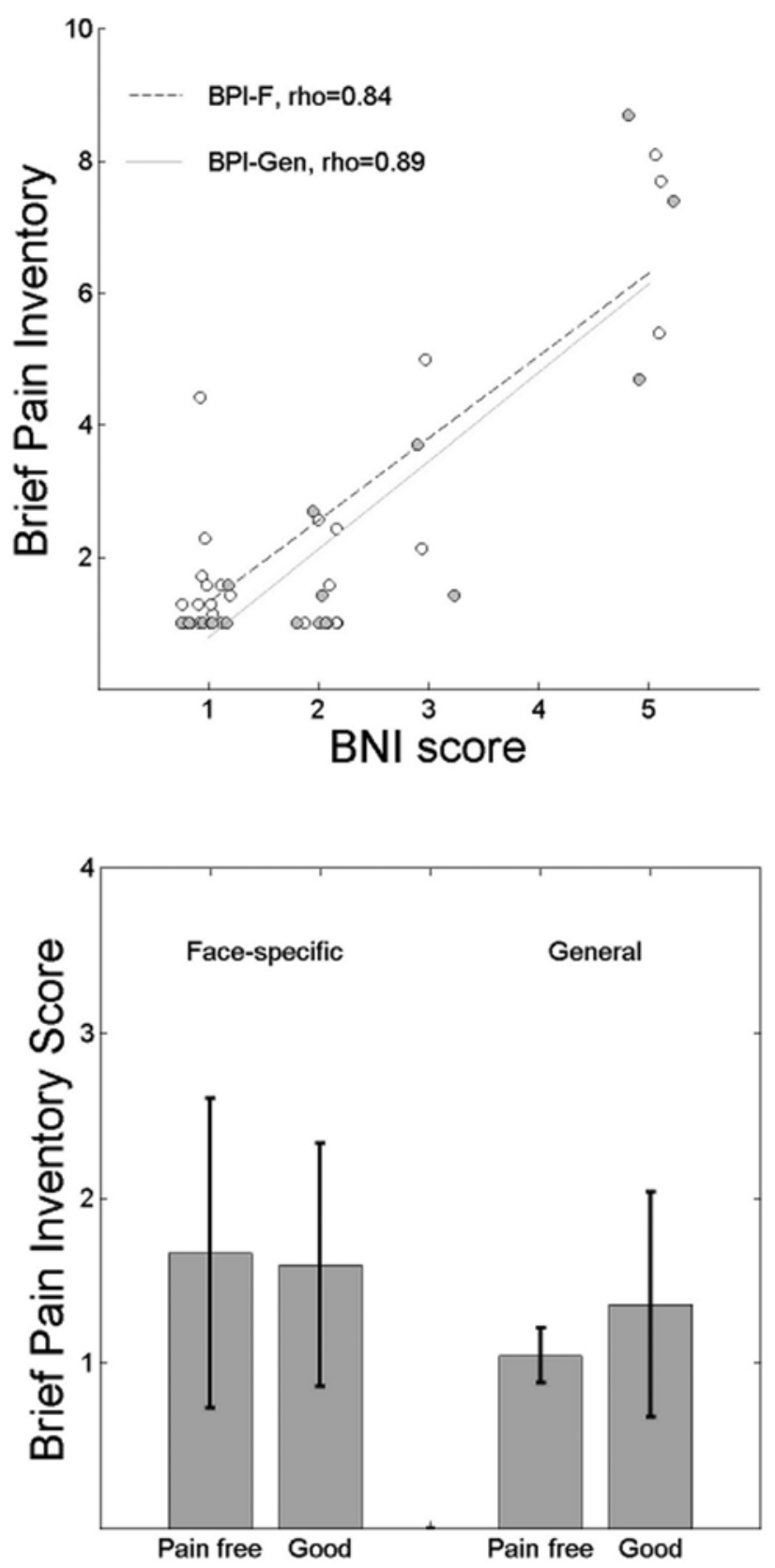

FIG. 4. Graphs of the impact of numbness after IN on QOL. Upper: $\mathrm{BPI}$ scores for impact on general activity and face-specific activity were highly correlated with BNI scores ( $p=0.00$ for BPI-Facial and $\mathrm{BPI}-G e n e r a l)$. A small amount of random noise was added to BNI scores to facilitate viewing of this plot; this was not used in statistical calculations. Lower: In patients with pain-free versus good outcomes, BPI scores were not significantly different $(p=0.14$, Student t-test, BPI-Facial; $p=0.48$, Student t-test, BPI-General). The implication is that numbness alone, or numbness with only occasional pain, does not greatly affect QOL, as both groups had mean and median BPI scores so close to 1 (no effect).

PSR report success rates of $50 \%$ at 2 years, ${ }^{3}$ and $60 \%$ at 5 years. ${ }^{22}$ The Kaplan-Meier curves in these studies are very similar to those reported here. Previous treatment for TN remains a poor prognostic factor as well. How IN compares to PSR awaits definitive evaluation in a larger 
number of patients. Our rationale for performing IN rather than PSR is 2-fold: first, by disrupting the nerve across its entirety, rather than only the lateral third or half, we anticipate disruption of a larger cross-section of nerve fibers that could be contributing to generation of triggered, lancinating pain; and second, the thought was to mitigate the severity of numbness, and impact on patient satisfaction, by leaving the nerve by and large in continuity.

Long-term pain-free results for IN and RFL appear to be similar in the short term. At 2-year follow-up, approximately $50 \%$ of patients undergoing RFL have experienced some pain recurrence. ${ }^{3,18}$ However, RFL shows a steady recurrence rate, while IN recurrence rates appear to drop after 2 years, and thus pain-free outcomes for IN appear more durable (Fig. 4). Pain-free survival for IN after 2 years resembles that of MVD; in other words, while RFL appears to have a steady decline in pain-free outcomes over time, early failure appears to be more likely for IN than late failure.

Comparison of IN with GKS results is somewhat problematic. Studies for stereotactic radiosurgery tend to consider pain controlled with medications a therapeutic success, in contrast to RFL and MVD, which generally report success as complete relief of pain. Moreover, different treatment paradigms in GKS make comparisons difficult. A recent systematic review of stereotactic radiosurgery for $\mathrm{TN}$ found that $60 \%$ and $50 \%$ of patients treated with GKS maintain complete pain relief on or off medications at 2 and 3 years, respectively. ${ }^{10}$ This is commensurate with our results for complete pain relief without medications. Compared with the results of a recent large GKS series, ${ }^{20} \mathrm{IN}$ appears to have a higher success rate throughout follow-up when compared with GKS (Fig. 5).

These comparisons are admittedly ad hoc. However, given that the patient population examined in this study is significantly younger than the "classic" patient with TN, it is clinically relevant. Ablative procedures such as GKS may be safe, but are less effective. RFL may have a similar effectiveness, but treatment success may be less durable. Intuitively, it would appear that IN is less destructive than PSR, in which nerve fibers are cut or coagulated. It remains to be seen whether this is the case, and whether there is a significant difference between the two in terms of the likelihood of developing deafferentation pain. We do have some evidence that subsequent treatment of recurrent TN pain after IN, with RFL, can be successful. Because of its durability, we suggest that IN may be the most appropriate initial treatment for younger patients with Type $1 \mathrm{TN}$ without NVC, with RFL held in reserve for recurrences.

\section{Postoperative Numbness and Dysesthesias}

Subjective numbness was present in almost all patients $(96 \%)$ to some degree after IN. This percentage is relatively high compared with the other reports on IN, which reported approximately a $15 \%$ rate of numbness. ${ }^{7}$ Reported rates of numbness after PSR range from $67 \%$ to $100 \% .^{17}$ The success of percutaneous procedures at relieving pain is predicated on producing some degree of sensory loss, and numbness or hypesthesia is generally limited in severity and well-tolerated by patients.,17

Our data indicate that the degree to which numbness
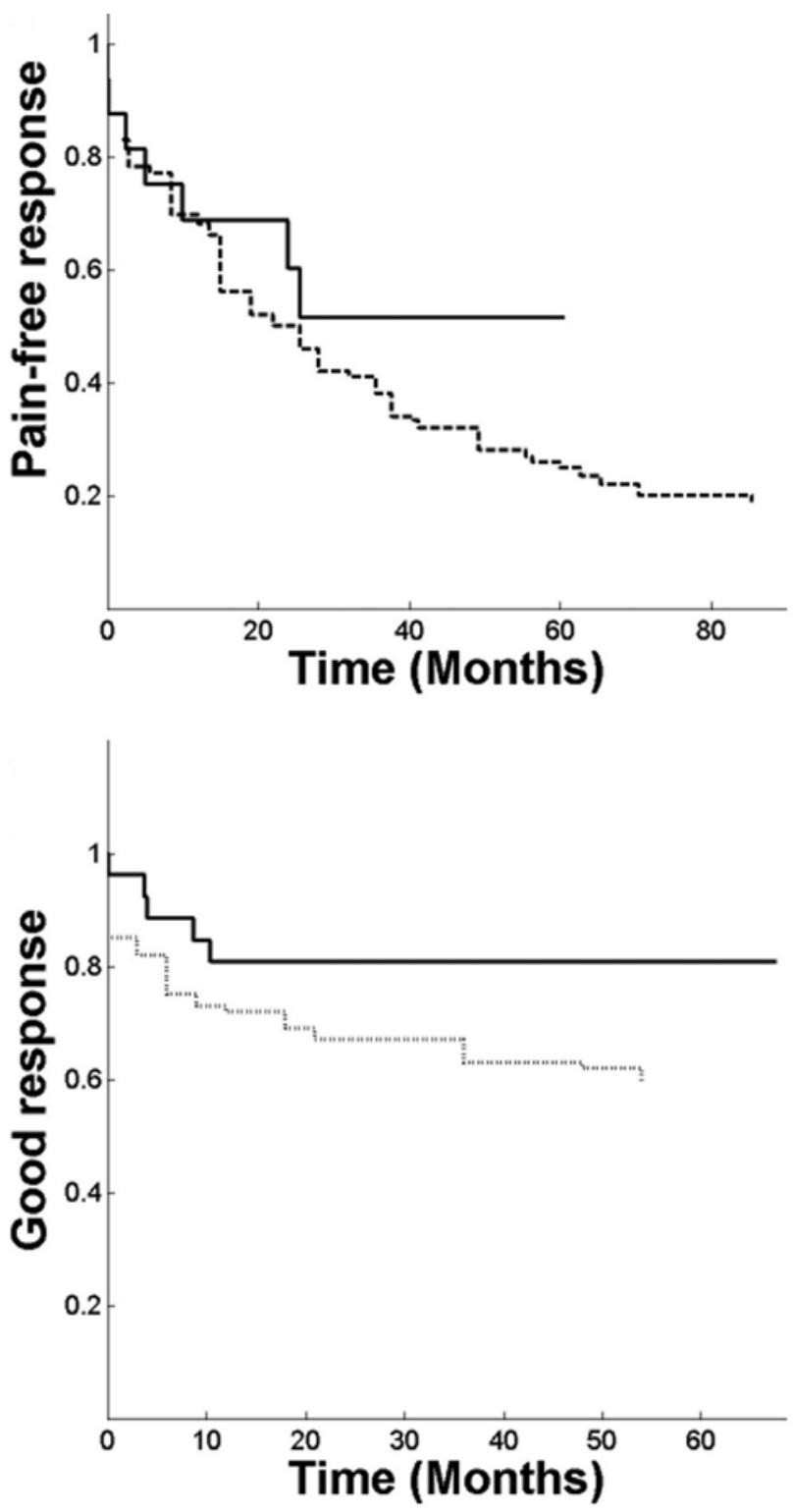

FIG. 5. Qualitative comparison of IN outcomes with RFL and GKS. Upper: Pain-free survival rates for IN patients (solid line) compared with those extracted from a large RFL study (dashed line).18 Initial recurrence rates up to 2 years appear nearly equivalent. However, after that point, recurrence rates for RFL appear to remain constant, whereas IN appears more durable. Lower: Failure rates extracted from a large GKS study (dotted line) versus IN (solid line). Treatment success in this plot is defined as in the GKS study, ${ }^{20}$ in which "adequate relief" with or without medication is considered a good outcome.

affects patient QOL is highly correlated to the presence and severity of pain. For our QOL measure, we sought to balance patient convenience with the desire for a validated measure. In particular, we sought to capture the effect of facial numbness on patient QOL. The BPI-Facial score meets that criterion, being fairly brief, while still capturing the impact of facial numbness on general activity and face-specific activity. The strong correlation between BPIFacial score and BNI pain score appears to indicate, however, that it is difficult to separate the effect of numbness from the effect of pain on patient QOL; it is very possible 
that the impact of pain versus numbness is conflated and cannot be separated one from the other.

A more thorough evaluation of sensory deficits induced by IN, including a preoperative assessment and objective measurement of hypesthesia, would clearly be beneficial in evaluating its side effects. Corneal numbness was not specifically evaluated, and given that IN as performed affects all 3 divisions of the trigeminal nerve intentionally, an assessment of the incidence of keratitis is likely warranted in the future. However, given the very low BPI-Facial scores for general and face-specific activities, it appears numbness in pain-free patients, or patients with good outcomes, is perceived as relatively minor.

A comparison of the effect on QOL brought on by numbness due to similar treatments for $\mathrm{TN}$ is an open question. Our impression is that numbness after IN does not significantly impact QOL in the majority of cases when pain is controlled; this outcome is likely similar for GKS and RFL. However, at this point, IN appears to be more durable than RFL, and more efficacious than GKS, which supports its use in a younger patient population. We have found that RFL is effective for pain control after treatment failure in IN, which supports the notion that further ablative procedures for TN can be effective after IN.

Several patients reported burning or aching pain in a trigeminal distribution after IN $(5 / 23,22 \%)$. At first glance, this appears to be an alarmingly high percentage of patients with deafferentation pain. However, all of these patients had prior treatments for TN. The majority of these patients (4/5) had some constant, aching pain prior to the IN procedure. We did not determine if this pain was better or worse than prior to IN.

There was 1 clear case of anesthesia dolorosa in a patient with no preoperative aching pain. This patient had a previous MVD for TN, and did present with a sensory deficit. After IN, they reported increased numbness, then burning pain in the same distribution that was refractory to medical treatment.

In conjunction with data on treatment failure rate over time, it is clear that previous treatment for TN poses a significant risk factor for adverse outcome when considering IN, whether it is treatment failure, deafferentation pain, or a more classic anesthesia dolorosa. This finding is borne out in many previous outcome studies for posterior fossa procedures for TN. Previous treatment with ablative (or nonablative, in the case of previous MVD) procedures is a risk factor for failure for MVD or PSR, and affects patient satisfaction rates after either surgery., ${ }^{1,211,22}$ Development of dysesthetic pain after posterior fossa exploration and MVD or PSR occurs in as many as $8 \%$ of patients during multiple repeat procedures for TN..$^{15}$ In this capacity, IN is no different from either MVD or PSR. Likewise, RFL carries a small risk of major dysesthesias, with incidence reports ranging from $0.9 \%$ to $10 \%$ in modern series. ${ }^{8,17,18}$ Interestingly, the largest study reports that the incidence of painful dysesthesia and anesthesia dolorosa did not correlate with the number of procedures performed. However, this may be confounded by the fact that the extent and degree of nerve damage is variable during RFL. Indeed, the rate of painful dysesthesia has been correlated with the extent of lesioning. ${ }^{17}$ As many as $15 \%$ of patients treated with GKS experience sensory loss impacting their QOL. Of note, GKS after a previous ablative procedure presents a similar increased risk for unpleasant numbness and deafferentation pain, with a 4-fold increase in incidence after a second treatment. ${ }^{10,20}$

Overall, like any ablative procedure, IN carries the risk of inducing unpleasant numbness, dysesthesias, and anesthesia dolorosa. In this small series, there was 1 patient with anesthesia dolorosa (4\%). The rate of dysesthesias or deafferentation pain is $16 \%$, but this may be confounded by previous treatments. Suffice it to say that IN, like any ablative procedure, is not an appropriate treatment for deafferentation pain and it remains to be determined whether it carries a significantly greater risk of causing deafferentation pain than any other destructive procedure.

\section{Limitations of the Study}

There are several obvious limitations to this study. It is a retrospective review. We do not have a control group, and historical comparisons to similar treatments are confounded by differing outcome measures and the lack of outcome measures reported over time. These factors exacerbate selection bias in our patient cohort. In general, during the period covered by this study, all patients without NVC were offered the option of posterior fossa exploration and IN. Nevertheless, additional bias is introduced during a retrospective study, when patients are not randomized to one treatment paradigm versus another. Factors such as patient age, or comorbidities, affect recommendations regarding the surgical plan offered to patients.

Also of note is that among this study population, 10 $(37 \%)$ of 27 patients endured a previous procedure for TN. Of those 10 patients, 7 (70\%) required further treatment for their TN. The study population appears to be inherently refractory to treatment for TN. This supports the notion that there is a factor outside NVC affecting incidence and recurrence of $\mathrm{TN}$; there may be a subgroup of patients who are refractory to treatment for $\mathrm{TN}$, regardless of the presence of NVC, or the patient population without NVC may be distinct from those with compression.

If anything, however, these questions should emphasize the efficacy and durability of IN as a treatment modality. A sizeable proportion of patients in this study are those who have proven refractory to previous treatments, and IN is nevertheless successful in many of these cases.

The sample size in this study is small, limiting the conclusions that can be drawn from statistical analysis. We sought to correct for this during statistical analysis, but the Yates correction in particular is known to be overly conservative. Also, while we reduced the number of factors examined in our Cox proportional hazards analysis, sample size is only barely adequate for this model. Nevertheless, the significant prognostic factors identified in this report are consistent throughout the TN literature.

We do not have a measure of baseline QOL prior to IN. While the BPI-Facial is internally consistent and valid, we do not have a control population against which to measure our results. Likewise, the lack of quantitative measures of sensory deficit makes it difficult to make conclusive statements about the production of numbness by IN.

However, the rate of follow-up for our limited popula- 
tion is excellent (96\%), with a significant portion of this follow-up lasting 3 years or more. The median follow-up duration is nearly 4 years, and thus the Kaplan-Meier survival tables are valid for more than 44 months. Despite its shortcomings, this study provides insight into the efficacy, durability, and value of IN as a treatment for TN when NVC is absent.

\section{Conclusions}

Internal neurolysis is an effective treatment for Type 1 TN when no NVC is present. It appears to be more effective than GKS, at least as effective as PSR, and is likely more durable than RFL. It is an ablative procedure, resulting in sensory deficit, and thus carries a higher risk of inducing deafferentation pain than MVD. Rates of painful dysesthesias do not appear to be inordinately higher than other ablative procedures, but evaluation of a larger patient sample is necessary. Prior treatment for TN is identified as a poor prognostic factor for pain relief, pain recurrence, and undesired side effects, which is shared with all other treatments for this condition. In patients who have not undergone prior treatment of TN and have no NVC on highresolution imaging, IN is an effective, safe, and durable treatment option. Particularly for young patients, IN may be the best treatment when NVC is not present given its durability. This is especially relevant because it appears that patients with Type $1 \mathrm{TN}$ without NVC are significantly younger than patients with Type $1 \mathrm{TN}$ and NVC.

\section{References}

1. Barker FG II, Jannetta PJ, Bissonette DJ, Larkins MV, Jho HD: The long-term outcome of microvascular decompression for trigeminal neuralgia. N Engl J Med 334:1077-1083, 1996

2. Bederson JB, Wilson CB: Evaluation of microvascular decompression and partial sensory rhizotomy in 252 cases of trigeminal neuralgia. J Neurosurg 71:359-367, 1989

3. Burchiel KJ, Steege TD, Howe JF, Loeser JD: Comparison of percutaneous radiofrequency gangliolysis and microvascular decompression for the surgical management of tic douloureux. Neurosurgery 9:111-119, 1981

4. Chen HI, Lee JYK: The measurement of pain in patients with trigeminal neuralgia. Clin Neurosurg 57:129-133, 2010

5. Headache Classification Committee of the International Headache Society: The International Classification of Headache Disorders, 3rd edition (beta version). Cephalalgia 33:629-808, 2013

6. Jannetta PJ: Arterial compression of the trigeminal nerve at the pons in patients with trigeminal neuralgia. J Neurosurg 26:159-162, 1967

7. Jie H, Xuanchen Z, Deheng L, Kun G, Fengyang X, Xiang C, et al: The long-term outcome of nerve combing for trigeminal neuralgia. Acta Neurochir (Wien) 155:1703-1708, 2013

8. Kanpolat Y, Savas A, Bekar A, Berk C: Percutaneous controlled radiofrequency trigeminal rhizotomy for the treatment of idiopathic trigeminal neuralgia: 25-year experience with 1,600 patients. Neurosurgery 48:524-534, 2001

9. Lee A, McCartney S, Burbidge C, Raslan AM, Burchiel KJ: Trigeminal neuralgia occurs and recurs in the absence of neurovascular compression. J Neurosurg 120:1048-1054, 2014
10. Lopez BC, Hamlyn PJ, Zakrzewska JM: Stereotactic radiosurgery for primary trigeminal neuralgia: state of the evidence and recommendations for future reports. J Neurol Neurosurg Psychiatry 75:1019-1024, 2004

11. Ma Z, Li M: "Nerve combing" for trigeminal neuralgia without vascular compression: report of 10 cases. Clin J Pain 25:44-47, 2009

12. McCartney S, Weltin M, Burchiel KJ: Use of an artificial neural network for diagnosis of facial pain syndromes: an update. Stereotact Funct Neurosurg 92:44-52, 2014

13. Miller JP, Acar F, Burchiel KJ: Classification of trigeminal neuralgia: clinical, therapeutic, and prognostic implications in a series of 144 patients undergoing microvascular decompression. J Neurosurg 111:1231-1234, 2009

14. Miller JP, Acar F, Hamilton BE, Burchiel KJ: Radiographic evaluation of trigeminal neurovascular compression in patients with and without trigeminal neuralgia. J Neurosurg 110:627-632, 2009

15. Pollock BE, Stein KJ: Surgical management of trigeminal neuralgia patients with recurrent or persistent pain despite three or more prior operations. World Neurosurg 73:523528,2010

16. Rogers CL, Shetter AG, Fiedler JA, Smith KA, Han PP, Speiser BL: Gamma knife radiosurgery for trigeminal neuralgia: the initial experience of The Barrow Neurological Institute. Int J Radiat Oncol Biol Phys 47:1013-1019, 2000

17. Taha JM, Tew JM Jr: Comparison of Surgical treatments for trigeminal neuralgia: reevaluation of radiofrequency rhizotomy. Neurosurgery 38:865-871, 1996

18. Tronnier VM, Rasche D, Hamer J, Kienle AL, Kunze S: Treatment of idiopathic trigeminal neuralgia: comparison of long-term outcome after radiofrequency rhizotomy and microvascular decompression. Neurosurgery 48:1261-1268, 2001

19. Tubbs RS, Griessenauer CJ, Hogan E, Loukas M, CohenGadol AA: Neural interconnections between portio minor and portio major at the porus trigeminus: application to failed surgical treatment of trigeminal neuralgia. Clin Anat 27:94-96, 2014

20. Verheul JB, Hanssens PE, Lie ST, Leenstra S, Piersma H, Beute GN: Gamma Knife surgery for trigeminal neuralgia: a review of 450 consecutive cases. J Neurosurg 113 Suppl:160-167, 2010

21. Young JN, Wilkins RH: Partial sensory trigeminal rhizotomy at the pons for trigeminal neuralgia. J Neurosurg 79:680687,1993

22. Zakrzewska JM, Lopez BC, Kim SE, Coakham HB: Patient reports of satisfaction after microvascular decompression and partial sensory rhizotomy for trigeminal neuralgia. Neurosurgery 56:1304-1312, 2005

\section{Author Contributions}

Conception and design: Ko, Burchiel. Acquisition of data: Ko, Ozpinar, Lee, Raslan. Analysis and interpretation of data: Ko, Lee, Raslan. Drafting the article: Ko. Critically revising the article: Burchiel, McCartney. Reviewed submitted version of manuscript: McCartney. Statistical analysis: Ko. Administrative/techni$\mathrm{cal} / \mathrm{material}$ support: McCartney. Study supervision: Burchiel.

\section{Correspondence}

Andrew L. Ko, Department of Neurological Surgery, Oregon Health \& Science University, CHH8N, 3303 SW Bond Ave., Portland, OR 97239. email: and@ohsu.edu. 